\title{
Ultra-wide band corrugated gaussian profiled horn antenna design
}

\author{
J. Teniente-Vallinas ${ }^{*}$, R. Gonzalo-García, C. del-Río-Bocio \\ Dept. of Electric and Electronic Engineering. Public University of Navarra \\ Campus de Arrosadia, s/n. 31006 Pamplona, (Navarra), Spain \\ Fax number: +34 948169720 Tfn. number: +34 948169326 \\ e-mail: jorge.teniente@unavarra.es,ramon@unavarra.es, carlos@unavarra.es
}

Abstract: A corrugated horn antenna design with more than $40 \%$ bandwidth is proposed in this paper. The measured radiated field patterns have a good agreement with the simulated ones.

This antenna can be used in applications that require extremely wide bandwidth with low sidelobes and low crosspolar levels, with not very strong return loss requirements.

\section{Introduction}

Nowadays, an increasing number of applications need stable radiation patterns with low sidelobes and low crosspolar levels in a very wide bandwidth. Gaussian horn antennas can offer one of the best solutions. As an example, it would be used as a feed for antenna measurements facilities; this kind of antennas could be used for very large bandwidth measurements, with the subsequent reduction in time and cost. Return loss of this antenna is not as good as the other parameters, but it could be improved.

\section{Antenna design}

The design was performed to acomplish an ultra-wide bandwidth, (more than $40 \%$ ), with low sidelobes and low crosspolar levels. The selected operational range of frequencies were in $\mathrm{X}$ and $\mathrm{Ku}$ bands. The goal was to connect to a WR-75 waveguide system, obtaining very good radiation features from 10 to $15 \mathrm{GHz}$.
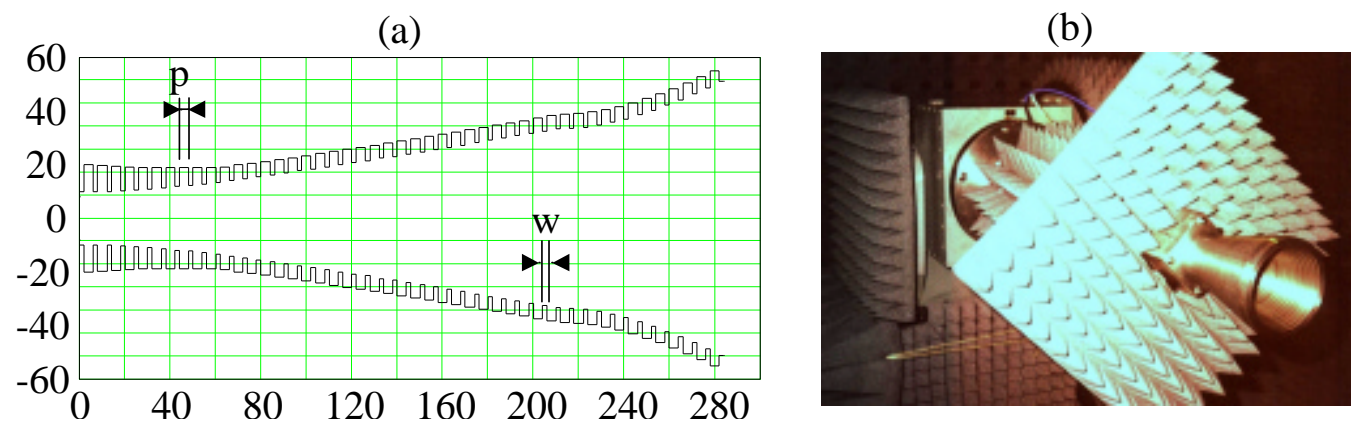

Fig. 1.- a) Corrugated horn antenna profile in mm b) Antenna mounted on the CATR

To design the horn antenna profile, gaussian tecniques were used. In fact the antenna is formed of two gaussian profiles stacked together (see fig. 1a).

A reduction of the manufacture cost was also considered as an important design parameter; therefore the corrugation period ( $p$ ) was fixed along the antenna at $6 \mathrm{~mm}$ while the tooth width (w) was $2 \mathrm{~mm}$ (see fig. 1a). 
The total length was $284 \mathrm{~mm}$, with an output radius of $59.6 \mathrm{~mm}$. The selected input radius was $11.6 \mathrm{~mm}$. At the throat, a waveguide transformer from $\lambda / 2$ to $\lambda / 4$ was used (see fig. 1 a).

\section{Manufacturing process}

Manufacturing process was made by TMM "Micromecanica S.A.", Oricain-Navarra in Spain. The antenna was fabricated in aluminium. The first part $(78 \mathrm{~mm})$ was done by means of stacked aluminium plates of the appropiate width. The rest of the antenna was made by a numerical milling machine and then all the parts were stacked together to obtain the final antenna design. A manufacture error of $0.1 \mathrm{~mm}$ was assumed along the antenna.

\section{Simulations}

A mode matching and scattering matrix numerical analysis was selected to design and simulate the behaviour of the antenna. Also the return loss tests were calculated using Ansoft HFSS finite element code, (see fig. 2a).

\section{Measurements}

Measurements were performed in ESA/ESTEC Compact Antenna Test Range. The CATR is an antenna measurement facility, based on a dual cylinder-parabolic reflector configuration installed in an environmentally controlled anechoic chamber. The reflectors design create a stable test zone which is large enough to measure the electrical characteristics of a wide variety of antennas.

To allow the wide bandwidth necessary to measure the antenna, two different feeds had to be used, the first was at WR-90 input waveguide which worked from 8.2 to $12.4 \mathrm{GHz}$, and the second at WR-62 input waveguide covering from 12.4 to $18 \mathrm{GHz}$.

The measurement of the proposed antenna was performed from 9 to $16 \mathrm{GHz}$ in steps of $5 \mathrm{MHz}$ with an azimuthal step of $1 \mathrm{deg}$, covering from -70 to $70 \mathrm{deg}$. E, $45 \mathrm{deg}, \mathrm{H}$ and $135 \mathrm{deg}$ planes were obtained in copolar polarization and $45 \mathrm{deg}$ and 135 deg planes also in crosspolar polarization for the whole band.

A very compact $(14 \mathrm{~mm})$ crosspolar free, rectangular to circular transition was used to feed the antenna from WR-75 waveguide standard to the $11.6 \mathrm{~mm}$ antenna input radius. The output radius of this transition was $9.5 \mathrm{~mm}$, and this was connected directly to the antenna. This transition and the step between the output of the transition and the input of the antenna modifies the return loss of the whole system, but not the radiation features, so the transition was considered good enough for our purposes.

Time gating was applied to obtain the antenna response from the multiple reflections of the chamber. Crosspolar levels below $-40 \mathrm{~dB}$ were difficult to measure due to the rectangular reflector configuration of the chamber and also due to the crosspolar level of the feeds. But it was considered acceptable, (see fig. 2 b).

\section{Comparisons between measurement and simulation.}

As it can be seen in figures $2 \mathrm{~b}$ and 3 , the antenna presents a measured maximum crosspolar level below $-30 \mathrm{~dB}$ from 9 to $14.8 \mathrm{GHz},(49 \%$ 
bandwidth), and below $-35 \mathrm{~dB}$ from 10.6 to $14.4 \mathrm{GHz}$, (more than $30 \%$ bandwidth). The return loss of the antenna is drawn in figure 2a. Values below $20 \mathrm{~dB}$ are obtained from 11 to $14 \mathrm{GHz}$, which can be considered good enough. Besides it can be improved by designing a better rectangular to circular transition.

The directivity of the antenna varies from 19 to $22 \mathrm{~dB}$ along the band.

(a)

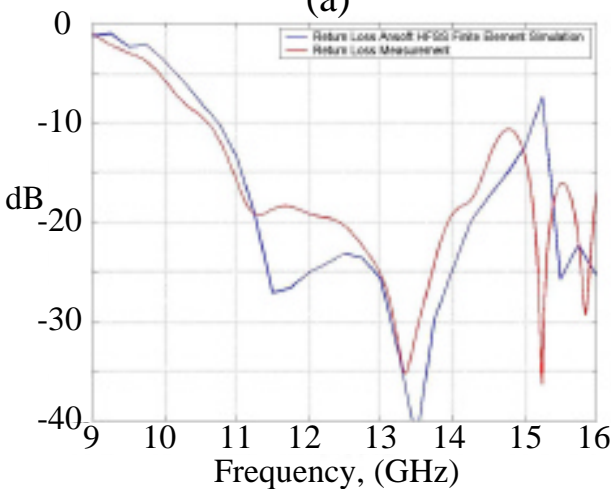

(b)

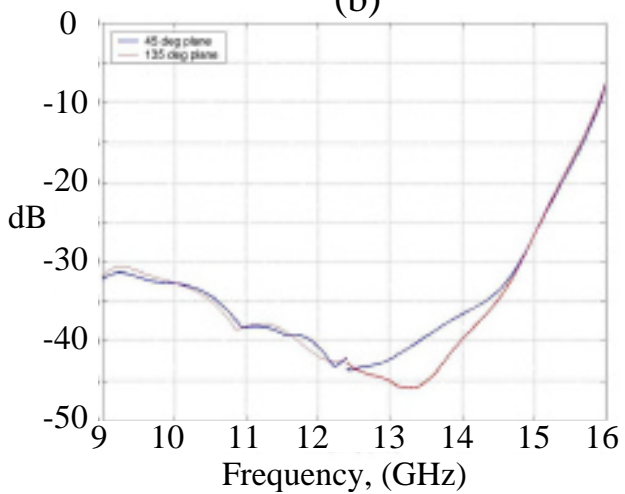

Fig. 2.- a)— Simulated Return Loss (Ansoft HFSS Finite Element Code)

- Measured Return Loss

b) Measured Crosspolar Maximum: -45 deg plane, —135 deg plane

\section{Conclusions}

An ultra-wide band corrugated horn antenna with low crosspolarisation and low sidelobes has been designed, manufactured and measured. Simulated and measured patterns show really good agreement.

This type of antenna is suitable for a wide variety of applications, i.e. as a feed for antenna measurement facilities.

Return loss is a critical parameter and should be improved in the future.

\section{References}

[1] J. Teniente, R. Gonzalo and C. del Río, "Gaussian Profiled Horn Antenna for Hispasat 1C Satellite", International Journal of Infrared and millimeter Waves, Vol. 20, N. 10, October 1999.

[2] J. Teniente, R. Gonzalo and C. del Río, "Gaussian Profiled Horn Antennas”, ISRAMT'99 $7^{\text {th }}$ International Symposium on Recent Advances in Microwave Technology. Malaga, Spain. December 1999.

[3] R. Gonzalo, C. del Río, J. Teniente and M. Sorolla, "New Approach to the Design of Corrugated Horn Antennas", $20^{\text {th }}$ ESTEC Antenna Workshop on Millimetre Wave Antenna Technology and Antenna Measurements, ESA/ESTEC Noordwijk, Holland. 18-20 of June, 1997.

[4] R. Gonzalo, C. del Río and M. Sorolla, "Optimal horn antenna design to excite high purity gaussian beam using overmoded waveguides" The XXI Int. Conference on Infrared and Millimeter Waves. Berlin, Germany. July 1996. 

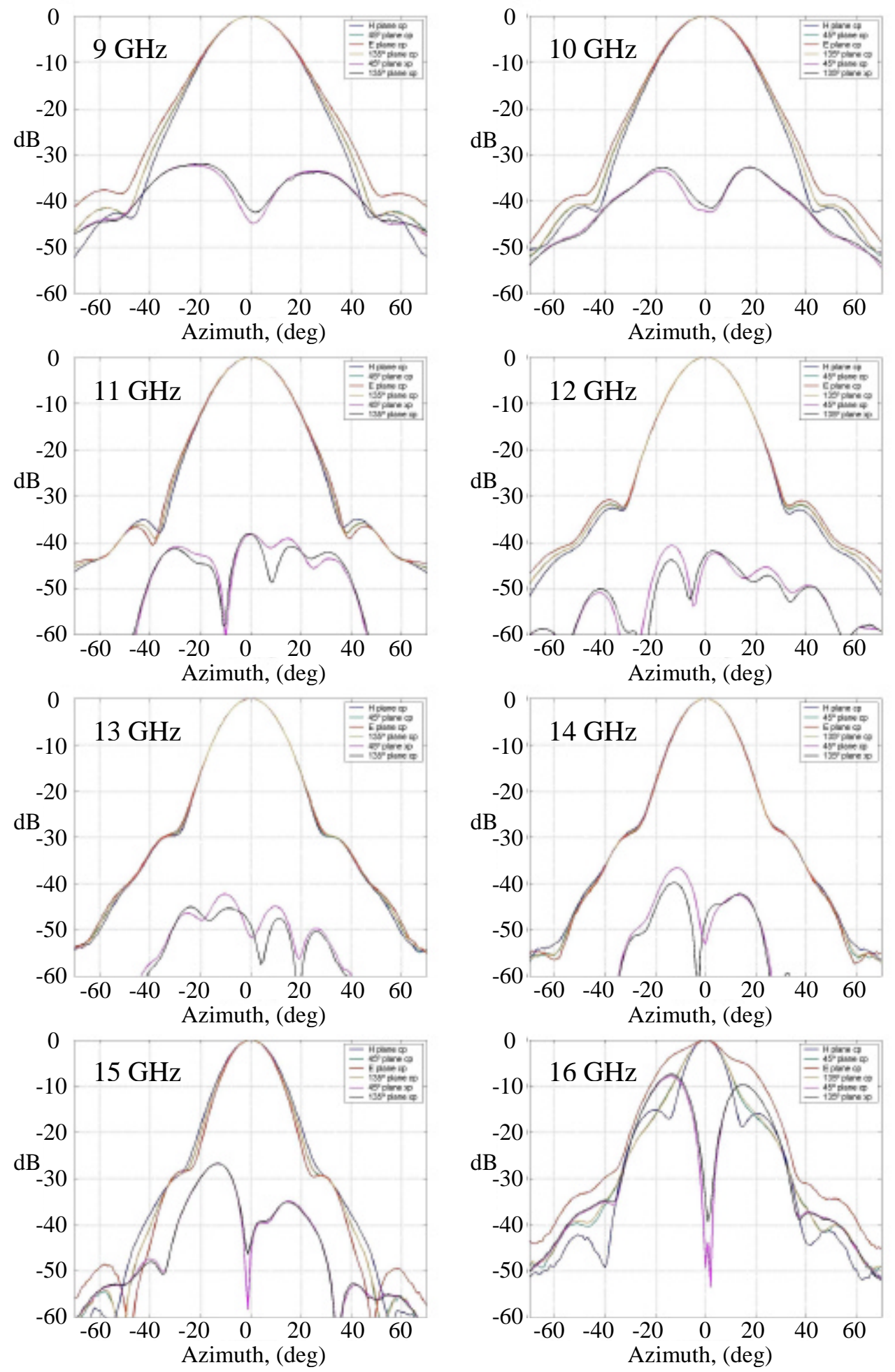

Fig. 3.- Measured far field radiation patterns.

H plane copolar, -45 deg plane copolar, $\_$E plane copolar
135 deg plane copolar, $\_-45$ deg plane crosspol, $\_135$ deg plane crosspol 